\title{
A Novel Reason Mining Algorithm to Analyze Public Sentiment Variations on Twitter and Facebook
}

\author{
Ashwini Patil \\ M.E Scholar \\ Computer Engineering \\ Department \\ Thakur College of Engineering \\ and Technology, Mumbai
}

\author{
R.R. Sedamkar \\ Professor \\ Computer Engineering \\ Department \\ Thakur College of Engineering \\ and Technology, Mumbai
}

\author{
Shiwani Gupta \\ Assistant Professor \\ Computer Engineering \\ Department \\ Thakur College of Engineering \\ and Technology, Mumbai
}

\begin{abstract}
With the explosive growth of social media on web, analyzing Public Sentiment Variations (PSV) has become utmost necessity as public sentiments fluctuate with alterations in real life events. Analysis of PSV empowers decision makers to gain a better understanding of public reactions in social, political and economic environment thus helps in better decision making. Most of the recent studies are bounded to be analyzing and predicting public sentiments. In this paper, we have done further analysis to know the useful insights for PSV using tweets and Facebook comments with a specific target. We propose a Novel Reason Mining Algorithm to find the possible reasons affecting PSV in significant sentiment variation period. It uses incremental iterative approach to refine the final list of most influential reasons. To give more intuitive representation, the algorithm ranks a set of mined reason candidates. The reason candidate with the most number of tweets/comments is the main reason for sentiment variation in that time period. The quality of mined reasons mainly depends on the quality of clusters formed prior to reason mining. The performance of our approach is compared with baseline K-means clustering technique. Experimental results show that our approach forms better clusters and effectively mine the reasons for sentiment variations. The Kmeans technique attained $57.05 \%$ precision and $53.31 \%$ recall on Twitter and $69.86 \%$ precision and $40.62 \%$ recall on Facebook. The Novel Reason Mining Algorithm achieved $79.4 \%$ precision and $44.45 \%$ recall on Twitter and $71.13 \%$ precision and $40.62 \%$ recall on Facebook.
\end{abstract}

\section{General Terms}

Data Mining, Sentiment Analysis.

\section{Keywords}

Public Sentiment Variation, Novel Reason Mining Algorithm, Twitter, Facebook

\section{INTRODUCTION}

Nowadays online social network become a prevalent means of people's social interaction. The social networking sites become ubiquitous and important component of individual's life. Per day, hundreds of millions of users share their emotions, thoughts, feelings and opinions on Twitter and Facebook. The vast textual information available on these sites is one of the major source for mining people's opinions and emotions, which will help make good decisions in different domains. Opinion mining and sentiment analysis is automated and computational study of recognising emotions and detecting polarity. These two fields use data mining and natural language processing (NLP) techniques to extract the knowledge from the source of information available on World Wide Web.

Prior sentiment analysis has faced difficulty in extracting sentiment label from long paragraphs, where variation in the document length made the task of classification very difficult. With the explosive growth of web applications, the means of people's communication have been changed from blogs and forums to Twitter and Facebook. Most of the recent studies have centered on Twitter and focused on analyzing and predicting public sentiment. However, very few research studies [1, 2] have been applied current sentiment analysis techniques on Facebook. Facebook also provides valuable information for analyzing public sentiment with 1.49 billion users contributing 3 million posts per minute. Unlike most of the studies, we tried to interpret public sentiment variations using tweets and Facebook comments. With time, the sentiments of people fluctuates reacting towards the real life events. These public sentiment variations can be analyzed to find possible reasons affecting on public sentiments. Such type of analysis can provide important information to make decisions in various aspects. For example, if public sentiment changes greatly on some product, the related companies may want to know why their products receive such feedback.

The main research goal of the proposed work is to design a system that will analyze the public sentiment variations on both Twitter and Facebook and mine possible reasons behind the variations. Interpreting public sentiment variations involves the process of tracking public sentiment variations about a specific target for example, "Narendra Modi," focusing on political domain. To track public sentiment variations, the tweets and comments regarding this target are classified into positive, negative and neutral. To classify the tweets/comments, Stanford NLP's Sentiment Analysis Tool is used.

On the basis of classification and using some descriptive statistics the public sentiment variations have been tracked. Inspiring from the work done by [3], we propose a Novel Reason Mining Algorithm that can mine the possible reasons behind the public sentiment variations. It uses incremental iterative approach to refine the final list of most influential reasons. To give more intuitive representation, the algorithm ranks a set of reason candidate. The reason candidates are ranked according to number of tweets/comments associated with the particular reason candidate.

The performance of the system has been evaluated on Twitter and Facebook datasets. The system is able to mine the 
possible reasons behind sentiment variations.

\section{RELATED WORK}

Sentiment analysis is very effective in many domains, as it provides the valuable information to make the critical decisions. Different from all the research studies, S.Tan, Yang Li, Huan Sun, Ziyu Cuan, Xifeng Yan, Jiajun Bu [3] moved one step further to investigate the problem of analyzing public sentiment variations on Twitter and finding the problem of possible reasons behind these variations. To solve the problem, they have proposed two LDA (Latent Dirichlet Allocation) based models FB-LDA and RCB-LDA. The task of interpreting public sentiment variation involves the process of sentiment analysis, event detection and tracking, data visualization, correlating tweets and events. The following section describes the related work done in these fields.

\subsection{Sentiment Analysis}

The substantial work has been done in the field of sentiment analysis over the past few years. Two approaches are widely used for opinion mining and sentiment analysis: 1) Machine learning based 2) Lexicon based.

The machine learning techniques became popular for sentiment analysis from the work done by Pang and Lee (2002) [4]. Three Supervised Classifiers: Naive Bayes (NB), Maximum Entropy (ME) and Support Vector Machines (SVM) were experimented on movie reviews collected from imdb.com. They compared the efficiency of these three classifiers with different feature selection methods. Their work was extended to improve the precision of NB [5]. They proposed subjectivity identification in the text as a preprocessing step and best accuracy up to $87.2 \%$ was achieved on the movie reviews dataset. The experiments with SVM, $\mathrm{NB}$ and ME on movie and car brand reviews by Boiy, E., Hens, P., Deschacht, K., and Moens, have also confirmed high performance of machine learning techniques with accuracy up to $90.25 \%$ [6]

An unsupervised learning algorithm for classifying reviews was proposed by Turney, in 2002 [7]. In that approach, first, adjectives and adverbs were extracted and then semantic orientation of extracted phrases was calculated using Pointwise Mutual Information (PMI). Finally reviews were classified as per average semantic orientation of the phrases. The experiments are conducted on different datasets like movie, bank and automobile. Positive and negative sentiment based summaries for product features from reviews (Amazon, CNET) were proposed by $\mathrm{Hu}$ and Liu (2004). They achieved $80 \%$ accuracy of the polarity classification in terms of precision [8]. In an attempt to assign sentiment scores to each distinct entity in the text and then assigning an overall subjectivity score to the text, Godbole et al. (2007) has proposed a sentiment lexicon based semantic approach [9]. Pang and Lee (2008), in their study gave the overview of the different approaches used in sentiment analysis [10].

Some researchers combined the machine learning and lexicon based approaches together to improve sentiment classification performance $[11,12]$. Both these techniques have their own pros and cons, but still the supervised machine learning techniques give more accuracy of sentiment classification than lexicon based techniques [13].

\subsection{Event Detection and Tracking}

The proposed work is mainly carried out to analyze the motives behind the public sentiment variations about a specific target. From the observations, we can say that events are good representatives of the reasons behind the public sentiment variations. The task of mining reasons, can be accomplished by detecting and tracking the events, and then analyzing the sentiments about each event and also tracking sentiment variations about the target and finding causes for the changes in sentiments.

Some existing event tracking methods are discussed here. Hila Becker [14] proposed a general model for identifying events in social media documents via clustering. They used similarity metric learning approaches to produce high quality clustering results. They experimented with ensemble and classification based techniques, combining a set of similarity metrics to predict when social media documents correspond to the same event. B-S Lee and J. Weng [15] worked on detecting events which are published on Twitter through analysis of the contents. They proposed EDCoW model that is Event Detection with Clustering of Wavelet based on Signals for detecting events. Leskovec et al. [16] proposed the method on tracking memes, for example quoted phrases and sentences. Their work offers the analysis of the global news cycle and the dynamics of information propagation between mainstream and social media.

All these methods are applicable for representative tweets such as the biggest event in the whole twitter message stream. The biggest disadvantage is that fine-grained events can be detected very hardly.

\subsection{Data Visualization}

The task of mining reasons is related to visualize the particular information hidden in the text data. Some researchers have worked on data visualization using subspace learning algorithms [17]. But data visualization techniques available in literature are not appropriate for noisy text data. Another visualization technique used widely is ranking.

D.Tao et.al [18] studied subspace learning algorithms and ranking. Retrieving images from large database is one of the emerging field today. Content based image retrieval (CBIR) technique is used to retrieve images from the large database. Though ranking is a core technique, and widely used in the field of information retrieval, it is not so much useful to solve the reason mining task.

\subsection{Correlation between Tweets and Events}

Some researchers $[19,20]$ have studied the correlation between events and tweets. Yuheng hu [19] proposed a joint statistical model ET-LDA that determines the topical influences between an event and its associated Twitter feeds (tweets). They claim that their work is the first step towards understanding complex interactions between events and social sites. Deepayan Chakrabarti and Punera [20] investigated the problem of real-time event summarization of tweets from twitter. They proposed a modified Hidden Markov Model that gives an intermediate representation for a sequence of tweets relevant for an event. SUMMHMM determines the hidden events and also works excellent on one-shot events, e.g. earthquakes.

\section{METODOLOGY}

The steps performed for analyzing public sentiment variations and mining the useful insights behind these variations are described in this section. First, the tweets and Facebook comments regarding specific target (e. g. "Narendra Modi") are extracted from the collected tweets and comments. The 
extracted tweets and comments are pre-processed to remove the noise in the data. The task of preprocessing is essential to make the sentiment analysis more efficient. Then these tweets/comments are classified into three classes like positive, negative and neutral by using Stanford NLP Classifier ${ }^{1}$. On the basis of classification of each tweet and comment, the sentiment variations regarding the specific targets have been tracked with the help of some descriptive statistics. Finally, the reasons behind these public sentiment variations have been analyzed by applying the Novel Reason Mining Algorithm. These steps are described in the following subsections.

\subsection{Extraction and Preprocessing of tweets and comments}

All the tweets and comments related to the specific target are extracted by filtering the tweets/comments which contain the keywords of the target. Generally the noise in the tweets and comments affects the results of sentiment analysis. The preprocessing techniques remove the noise and make them efficient for sentiment classification. The preprocessing techniques used in this work are described here in the following subsections.

\subsubsection{Stop Word Removal}

Extremely common words are not considered in order to speed up results. These filtered words are known as 'Stop Words'. A list of commonly used stop words is retained and it is used to remove all the stop words from tweets/comments.

\subsubsection{Stemming}

Stemming is done for reducing inflected (or sometimes derived) words to their stem, base or root form. For example the word "Happiness", indicate the positive sentiment. To extract the correct sentiment from these type of words. They should be stemmed to its root i.e. the word "Happy". A popular Porter stemmer algorithm is used for stemming process [21].

\subsubsection{Conversion of Slang Words}

User Tweets and comments often contain slang words. The slang words like omg, lol play important role in sentiment analysis. A list of commonly used slang words is maintained along with their translation in standard form. The list is created by using Internet Slang Word Dictionary ${ }^{2}$. First we converted the slang words into their standard forms and then used them in tweets/comments to analyze the sentiment.

\subsubsection{Removal of URL}

Most of the tweets and comments contain URLs. These URLs should be removed to make the task of sentiment analysis more effective. To remove the URLs a regular expression containing a pattern for URL is used. The string matching with the pattern is removed from the tweets/comments.

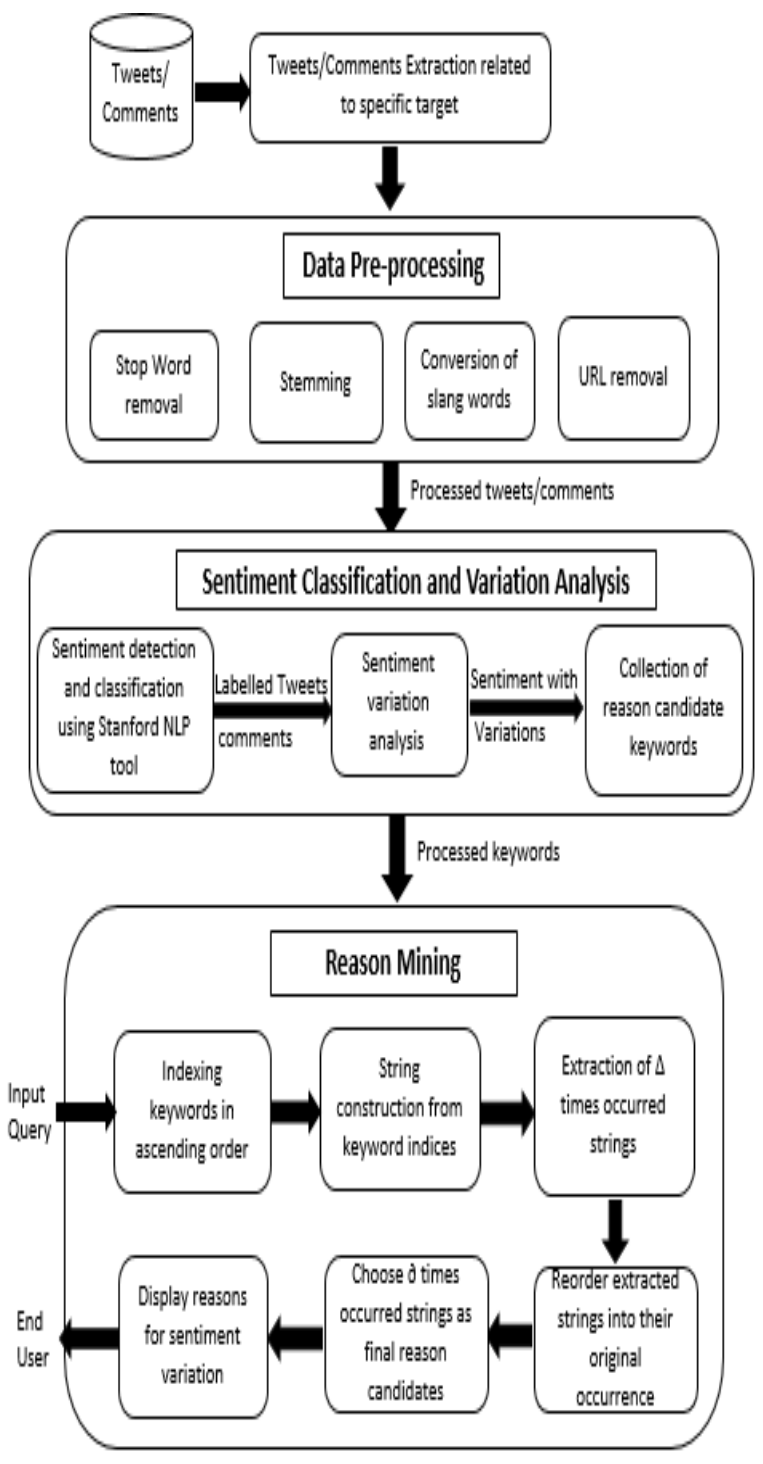

Fig 1: Methodology Flowchart

\subsection{Sentiment Classification}

To classify the tweets and comments as positive, negative and neutral, a sentiment analysis tool of Stanford NLP framework ${ }^{1}$ is used. This tool is based on principle of Maximum Entropy Classifier, which classifies the tweets/comments into positive, negative and neutral classes. This Stanford NLP tool has proven its quality and accuracy, as various sentiment processing applications has adopted it as a popular sentiment analysis tool in machine learning. Thus we can estimate the probability that an opinion may contain positive, negative or neutral sentiments.

\subsection{Sentiment Variation Analysis}

After classifying all the extracted tweets and comments about a target, we analyzed the sentiment variations using the number of positive and negative tweets/comments. But the number of positive and negative tweets is not so useful, as this number may change consistently. In this work, we considered percentage of positive, negative and neutral tweets/comments to analyze the sentiment variations, e.g. the percentage of negative/positive tweets/comments is increasing more than $50 \%$. The statistics obtained after classification is the useful 
indicator for sentiment variation over the period of time.

The following figures show the public sentiment variations towards "Narendra Modi" on Twitter and Facebook from January, 2015 to April, 2015.

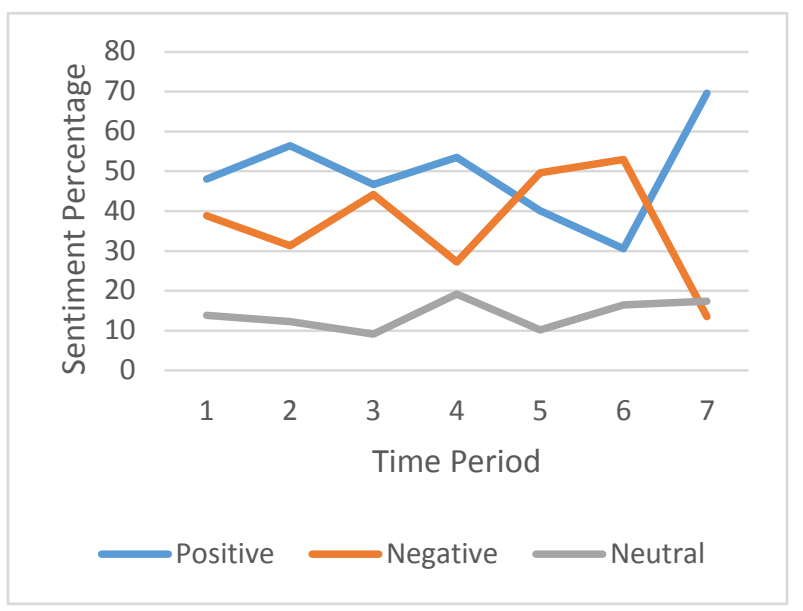

Fig 2: Public Sentiment Variation towards "Narendra Modi" on Twitter

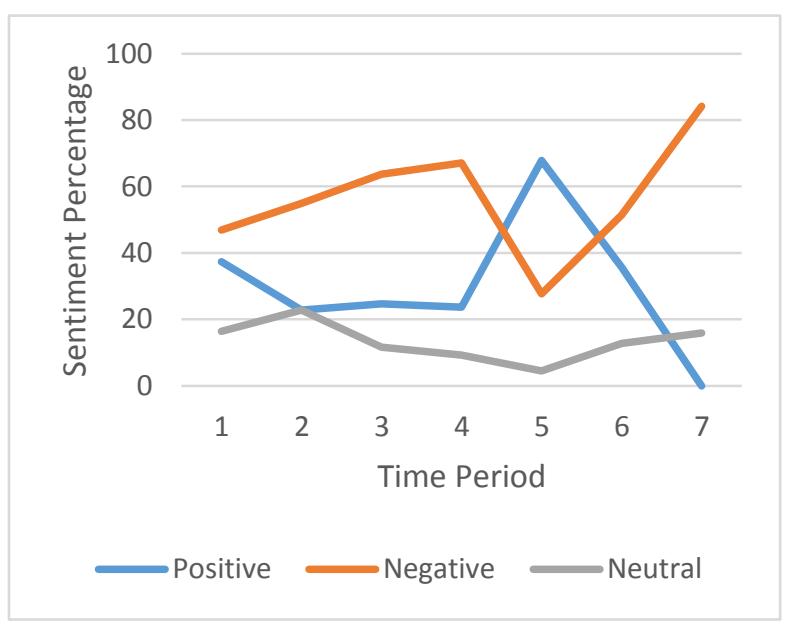

Fig 3: Public Sentiment Variation towards "Narendra Modi" on Facebook

\subsection{Reason Mining}

Reasons are the core part of tweet/comment, which is actually dominating factor of our current context. Actually finding reasons is not an easy task, but we can find clues by analyzing the tweets/comments in variation period. People give their opinion based on current reason for particular entity e.g. person, organization, event, etc. A Novel Reason Mining Algorithm is implemented to specify list of reasons within specific time interval for particular entity. Before applying this algorithm, all the keywords (uncommon words) of a tweet/comment have been separately stored in a table to make processing easier. A separate list of the most common occurred words during specific time interval is created. These most commonly occurred words are sub-parts of our main reasons to be mined. Then the association relationship between these most commonly occurred words and their combined occurrence count is determined. This combined occurrence count will gave us sorted list of main reasons.

Terms and Notations:
We used following notations in algorithm to represent main entities to be considered in calculations.

$\mathrm{W}$ : time window of specific days

$\mathrm{T}$ : a set of user tweets within time window $\mathrm{W}$

$\Delta$ : threshold value to be used to consider association occurrence count between keywords

$\partial:$ min value to be used to consider sequence occurrence count between keywords

Algorithm:

1. A set of user Tweets within the time window ' $\mathrm{W}$ ' are extracted from database.

2. In processing stage we already stored all the keywords of tweets in separate table. We take most occurred keywords within current time window ' $\mathrm{W}$ ' and store in separate array in ascending order.

3. A string is constructed from array index of keywords.

For example, consider the following array of most commonly occurred keywords within specific window [government, Dubai, India, minister, prime, Indian, world, people, vote]

A sample tweet is shown below:

Modi IN Dubai. It's Respectable Modi that can changed the Indian scenario toward world power.

This tweet contains three words from above array of keywords (Dubai, Indian, people). Then based on their indices " $1-5-7$ " string is constructed.

4. A string of array index is finally considered if more than $\Delta$ (threshold value) number of tweets produces such string.

5. A set of string of array index is then sorted in descending order based on their occurrence counts.

6. This set of string of array index is again processed to construct string of keywords which is final reason.

7. Actually this reason is a sorted string. So to achieve exact ordered reason based on its exact sequence again it is compared with tweets.

8. $\partial$ (min value) times occurred word sequence is finally shown as mined reason.

\section{RESULTS AND DISCUSSIONS}

The results are drawn by executing the system on real Twitter and Facebook datasets for mining possible insights behind public sentiment variations. The experimental results show that our system works well on both Twitter and Facebook. The Twitter and Facebook datasets are created manually to evaluate the system. We chose a target "Narendra Modi" as representative of political domain to evaluate the effectiveness of the system. The analysis of sentiment variation in this domain helps the politicians for decision making and reverting public opinion. The dataset spans from January 11, 2015 to April 1, 2015.

The performance of the system is evaluated on around 500 Twitter tweets and 500 Facebook comments. The Twitter Dataset and Facebook Dataset are processed in two steps: Preprocessing and Sentiment Classification. In Pre-processing stage, all the stop words are removed and remaining words are 
brought to their base forms using stemming. In sentiment classification the entire tweet's and comment's sentiment label is decided. A time window of 10 days is considered for tracking variations. Figures 2 and 3 show the public sentiment variation towards "Narendra Modi" on Twitter and Facebook respectively.

To evaluate accuracy of sentiment classification, the Twitter tweets and Facebook comments are manually labelled as positive, negative and neutral. The accuracy of the system is measured against the ground truth of the sentiment labels for each tweet/comment. The Overall accuracy is measured in terms of precision and recall. The number of tweets/comments varied for each time window. The performance of the Stanford's NLP classifier is evaluated on Twitter and Facebook. The following figure shows the precision and recall curves for Stanford's NLP classifier on Twitter and Facebook datasets. This classifier achieved $60.85 \%$ accuracy on Twitter dataset and $62.22 \%$ accuracy on Facebook dataset.

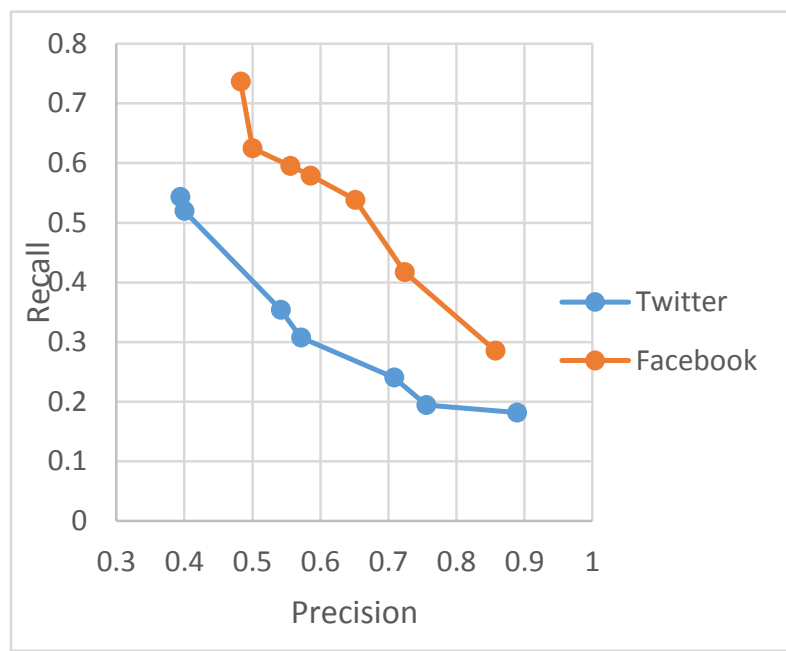

Fig 4: Precision-Recall curves for Classifier on Twitter and Facebook

The task of reason mining depends on how accurately the tweets/comments have been classified. If the accuracy of the classification is low, it may affect the final result of reason mining. For example, the reason candidates mined for positive sentiment variation may contain negative and neutral tweets. Today's sentiment classification techniques are not so much effective on the data like tweets/comments, as these data contains so much noise. Our approach has still achieved the satisfactory results of the classification on both Twitter and Facebook.

In this work, the percentage of positive, negative and neutral tweets/comments is considered to track the sentiment variations. We adopted the strategy that if the percentage of positive sentiments is more than that of negative in the particular time period, we mine the possible reasons behind the positive sentiment variation and vice-a-versa. To find the variation period, we considered the percentage of negative or positive tweets/comments is increasing more than $50 \%$. The reasons mined for the variation are ranked according to the number of tweets/comments associated with that reason candidate. The reason with the highest count will be the main cause behind the sentiment variation.

Example:
TABLE 1. Twitter: Negative sentiment variation towards "Narendra Modi" from February 20th to March 2nd.

\begin{tabular}{|l|c|c|}
\hline \multicolumn{1}{|c|}{ Reason Candidate } & Total Count & $\begin{array}{c}\text { Relevant } \\
\text { Count }\end{array}$ \\
\hline Modi government & 21 & 9 \\
\hline Modi skill development india & 8 & 5 \\
\hline
\end{tabular}

TABLE 2. Facebook: Positive sentiment variation towards "Narendra Modi" from March 13th to March 23rd

\begin{tabular}{|l|c|c|}
\hline \multicolumn{1}{|c|}{ Reason Candidate } & Total Count & $\begin{array}{c}\text { Relevant } \\
\text { Count }\end{array}$ \\
\hline $\begin{array}{l}\text { Modi bring internet indian } \\
\text { google prime minister }\end{array}$ & 16 & 12 \\
\hline $\begin{array}{l}\text { Modi bring internet indian } \\
\text { google }\end{array}$ & 8 & 5 \\
\hline
\end{tabular}

The above reason ranking example shows the public sentiment variation towards "Narendra Modi" for a specific time period. In this example, "Modi government" is the main reason candidate for negative sentiment variation, while "Modi bring internet india" is the main reason candidate for positive sentiment variation on Twitter and Facebook respectively. The total count gives the number of total tweets/comments associated with the reason candidate and relevant count gives the number of tweets/comments mined accordingly for positive/negative variation. For example, if there is negative variation, the relevant count is the number of negative tweets/comments associated with the reason candidate. The mined reason candidates may look like less informative for the sentiment variation. To get the more knowledge, anyone can see the actual tweets/comments that are linked with the reason candidate.

To check the efficiency of the reason mining algorithm, the ratio of the number of reason candidates mined against the total number of tweets/comments in the specific time period is computed. The number of mined reason candidates varies per time window. The ratio is quite steady for twitter tweets due to its size limit of characters and length of sentences present in it. The Facebook comments have more flexibility over its character size and number of sentences per comment. It produces more confusion to mining algorithm, making mining ratio more variable for Facebook comments. The following figure shows the retrieval rate of reason candidates per time window on Twitter and Facebook.

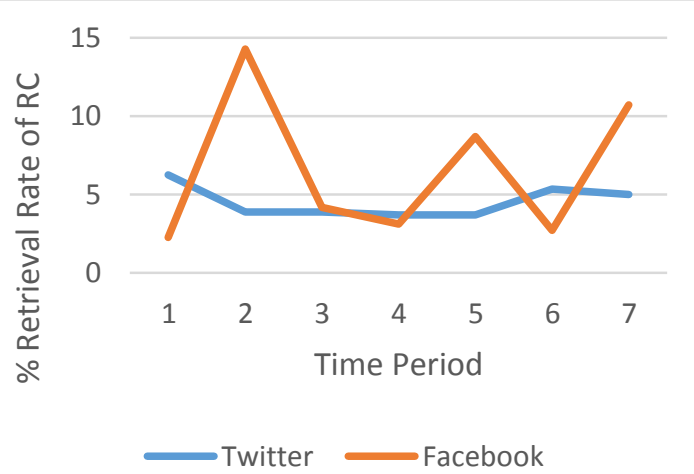

Fig 5: Retrieval Rate of Reason Candidate for Twitter and Facebook

The quality of mined reasons mainly depends on the quality of clusters formed before actual reason mining task. To verify 
that the Novel Reason Mining Algorithm effectively forms clusters of identical tweets and comments, we first manually checked the ground truth. The text clustering is done on the tweets/comments for each time period. Then precision and recall values are evaluated for Novel Reason Mining Algorithm. A baseline algorithm, K-means is designed to compare the efficiency of our algorithm in forming clusters. Experimental results show that our approach forms better clusters and effectively mine the reasons for sentiment variations. We have also compared the performance of the system on Twitter and Facebook, as these are two different platforms. The K-means technique attained $57.05 \%$ precision and $53.31 \%$ recall on Twitter and $69.86 \%$ precision and $40.62 \%$ recall on Facebook. The Novel Reason Mining Algorithm achieved the $79.4 \%$ precision and $44.45 \%$ recall on Twitter and $71.13 \%$ precision and $40.62 \%$ recall on Facebook. The following figures show the precision-recall curves for Novel Reason Mining Algorithm and K-means on Twitter and Facebook respectively.

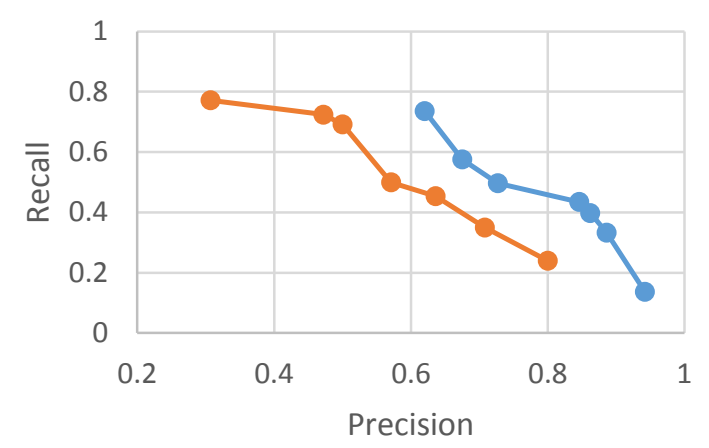

- Novel Reason Mining Algorithm - $\mathrm{K}$ means

Fig 6: Precision-Recall curves for Novel Reason Mining Algorithm and K- means on Twitter

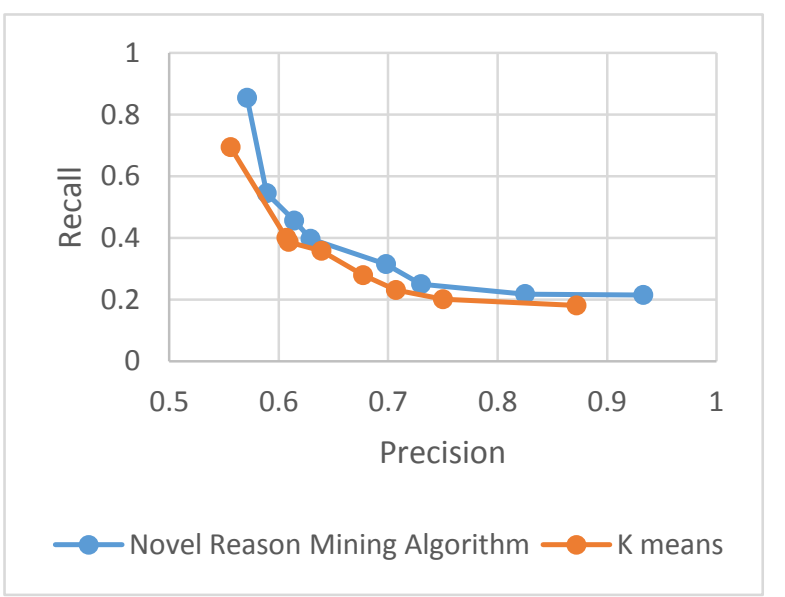

Fig 7: Precision-Recall curves for Novel Reason Mining Algorithm and K-means on Facebook

\section{CONCLUSION}

This paper focuses on analyzing the public sentiment variations and mining possible reasons behind these variations on Twitter and Facebook. To track the public sentiment, the Stanford NLP's sentiment analysis tool is used, which classifies the tweets/posts into positive, negative and neutral classes. On the basis of classification, the public sentiment variations have been tracked with the help of some descriptive statistics. The Novel Reason Mining Algorithm can find the possible reasons behind public sentiment variations. It uses incremental iterative approach to refine the final list of most influential reasons. To give more intuitive representation, the set of mined reasons are ranked according to the number of tweets/comments associated with the particular reason. The reason candidate with the most number of tweets/comments is the main reason for the sentiment variation in that time period. Our experimental results show that our approach is able to achieve $60.85 \%$ accuracy of sentiment classification on Twitter and $62.22 \%$ accuracy on Facebook. The quality of mined reasons mainly depends on the quality of clusters formed before mining the reasons. The performance of our method is compared with $\mathrm{K}$-means clustering technique. Experimental results show that our approach forms better clusters and effectively mine the reasons for sentiment variations. The performance of the system is also compared on Twitter and Facebook, as these are two different platforms. The K-means technique attained $57.05 \%$ precision and $53.31 \%$ recall on Twitter and $69.86 \%$ precision and $40.62 \%$ recall on Facebook. The Novel Reason Mining Algorithm achieved the $79.4 \%$ precision and $44.45 \%$ recall on Twitter and $71.13 \%$ precision and $40.62 \%$ recall on Facebook. The work is tested on small size dataset with 500 tweets and 500 comments. We conclude that our approach works better with small size dataset, irrespective of character limitation on Twitter, which is 140 characters. For Facebook there is no such limitation on characters for a single post. Previous research studies focused on Twitter for analyzing public sentiment variations. In this paper we tried to analyze public sentiment variations on Facebook also. In future, we will work on large datasets. The efficiency of the system will be checked on different platforms like movie and product reviews, blogs and forums.

\section{REFERENCES}

[1] J. Ahktar, S. Soria, "Sentiment Analysis: Facebook Status Messages", Stanford University, 2009

[2] Alvaro Ortigosa, Jose M.Martin, Rosa Carro, "Sentiment Analysis in Facebook and its application to e-learning" in proceedings of Computers in Human Behavior 31,527541, Elsevier, 2014.

[3] Shulong Tan, Yang Li, Huan Sun, Ziyu Guan, XifengYan, Jiajun $\mathrm{Bu}$, , Chun Chen, , and Xiaofei He,IEEE Transactions on "Interpreting the Public Sentiment Variations on Twitter," VOL. 26, NO. 5, MAY 2014

[4] B. Pang, L. Lee, and S. Vaithyanathan, "Thumbs up? Sentiment classification using machine learning techniques," Proceedings of the ACL-02 conference on Empirical methods in natural language processing, vol.10, 2002, pp. 79-86.

[5] Pang B., Lee, L., "A sentimental education: sentiment analysis using subjectivity summarization based on minimum cuts",Proc. Of 42nd annual meeting of the association for Computational linguistics(ACL), 2004, 271-278

[6] Boiy, E., Hens, P., Deschacht, K., and Moens, M.F. "Automatic sentiment analysis in on-line text" Proceedings Of 11th International Conference on Electronic Publishing(Vienna, Ausrtria) 2007 
[7] P. Turney, "Thumbs up or thumbs down? Semantic orientation applied to unsupervised classification of reviews", Proceedings of the Association for Computational Linguistics (ACL), 2002, pp. 417-424.

[8] M. Hu and B. Liu, "Mining and summarizing customer reviews," Proceedings of the tenth ACM international conference on Knowledge discovery and data mining, Seattle, 2004, pp. 168-177.

[9] Godbole,N., Srinivasaiah, M.Skiena S. "Large scale sentiment analysis for news and blogs", Proceedings of the International Conference on Weblogs and Social media (ICWSm'07), 2007

[10] B. Pang and L. Lee, "Opinion mining and sentiment analysis," Foundations and Trends in Information Retrieval 2(1-2), 2008, pp. 1-115.

[11] Anuj Sharma, Shubhamoy Dey "Artificial Neural Network Based Approach for Sentiment Analysis of Opinionated Text”, ACM transaction, 2012

[12] A. Mudinas, D. Zhang, M. Levene, "Combining lexicon and learning based approaches for conceptlevel sentiment analysis", Proceedings of the First International Workshop on Issues of Sentiment Discovery and Opinion Mining, ACM, New York,NY, USA, Article 5, pp. 1-8, 2012

[13] Ashwini Patil, Shiwani Gupta, "A Review on Sentiment Analysis Approaches" Multicon-W 2015 (ICWCCV 2015), vol 1, pp.243-247, ISBN: 978-0-9884925-7-8, February 2015
[14] H. Becker, M. Naaman, and L. Gravano, "Learning similarity metrics for event identification in social media," in Proc. 3rd ACM WSDM, Macau, China, 2010.

[15] J. Weng and B.-S. Lee, "Event detection in twitter," in Proc. 5thInt. AAAI Conf. Weblogs Social Media, Barcelona, Spain, 2011.

[16] J. Leskovec, L. Backstrom, and J. Kleinberg, "Memetracking and the dynamics of the news cycle," in Proc. 15th ACM SIGKDD, Paris, France, 2009.

[17] Kunpeng Zhang, Yu Cheng, Yusheng Xie, Ankit Agrawal, Diana Palsetia, Kathy Lee, and Alok Choudhary, "SES: Sentiment Elicitation System for Social Media Data", ICDM-SENTIRE 2011

[18] D. Tao, X. Tang, X. Li, and X. Wu, "Asymmetric bagging and random subspace for support vector machines-based relevance feedback in image retrieval", IEEE Trans. Patt. Anal. Mach. Intell.,vol. 28, no. 7, pp. 1088-1099, Jul. 2006

[19] Y. Hu, A. John, F. Wang, and D. D. Seligmann, "Et-lda: Joint topic modeling for aligning events and their twitter feedback," in Proc.26th AAAI Conf. Artif. Intell, Vancouver, BC, Canada, 2012.

[20] D. Chakrabarti and K. Punera, "Event summarization using tweets", in Proc. 5th Int. AAAI Conf. Weblogs Social Media, Barcelona, Spain, 2011.

[21] M.F. Porter, "An algorithm for suffix stripping", Program, Vol. 40, Issue: 3, 2006, pp.211-218 